\title{
Gestão do conhecimento na educação ambiental: estudo de casos em programas de educação ambiental em universidades brasileiras
}

Giovana Escrivão

\begin{abstract}
Graduada em Administração pela Faculdade de Ciências e Letras de Araraquara da Universidade Estadual Paulista "Júlio de Mesquita Filho" . Mestre em Engenharia de Produção pela Escola de Engenharia de São Carlos,Universidade do Estado de São Paulo - USP. Doutoranda em Engenharia de Produção pela Universidade Federal de São Carlos.
\end{abstract}

Marcelo Nagano

Mestre e Doutor em Engenharia Mecânica pela Escola de Engenharia de São Carlos da Universidade de São Paulo, Pós-doutor em Matemática e Computação Aplicada pelo Laboratório Associado de Computação e Matemática Aplicada do Instituto Nacional de Pesquisas Espaciais. Pesquisador e Bolsista de Produtividade e Pesquisa do CNPq. Professor dos programas de Graduação e Pós-graduação em Engenharia de Produção da Escola de Engenharia de São Carlos Universidade de São Paulo.

http://dx.doi.org/10.1590/1981-5344/1602

A importância da gestão do conhecimento para qualquer ambiente organizacional como uma estratégia de enfrentamento à instabilidade atual é amplamente reconhecida, apesar disso, pouco se sabe sobre a criação do conhecimento organizacional. Diversos são os contextos em que a gestão do conhecimento pode ser aplicada proporcionando a geração de novos conhecimentos, sendo um deles a educação ambiental, diante da crescente degradação ambiental. O objetivo dessa pesquisa é analisar e descrever o processo de criação do conhecimento em programas de educação ambiental. A pesquisa tem natureza qualitativa e caráter descritivo e pode ser definida como estudo de casos, pois foi realizado um estudo aprofundado das características dos programas de educação ambiental em duas das principais universidades brasileiras. Os métodos utilizados 
para coletar e analisar os dados foram a entrevista e a análise de conteúdo. Ao final da pesquisa pôde-se sugerir práticas aos programas de educação ambiental que podem proporcionar a gestão e estimular a criação de novos conhecimentos, como por exemplo, substituir as equipes, que são atualmente homogêneas, por equipes heterogêneas para que diferentes contextos, experiências e conhecimentos sejam compartilhados. No que diz respeito a teoria da criação de conhecimento, os casos estudados possibilitaram a identificação de observações sobre alguns pressupostas desta teoria, como por exemplo, a alta rotatividade dos alunos e a estabilidade garantida dos funcionários influem sobre 0 comprometimento destes com a criação de conhecimento.

Palavras-chave: Gestão do conhecimento. Criação do conhecimento. Conhecimento. Informação. Educação ambiental. Programas de educação ambiental. Universidades.

\section{Knowledge management in environmental education:case studies in environmental education programs in Brazilian universities}

Although the importance of knowledge management for any organizational environment as a strategy to confront the current instability has been widely recognized, little is known concerning the creation of organizational knowledge. Different contexts that knowledge management can be applied providing creation of new knowledge and its better use, like in the environmental education on accounting of growing environmental degradation. The present paper analyzes and describes the process of knowledge creation in environmental education programs at the two largest Brazilians public universities by conducting comparative case studies. This research can be classified as qualitative with a descriptive characteristic conducted through case studies, as a detailed study of the characteristics of the environmental education programs was realized in two major Brazilian universities. The methods used to collect and analyze the data were interviews and content analysis. At the end of the study it was made some suggestions of practices that 
can provide manage of the environmental knowledge and incentive for the creation of new knowledge to environmental education programs, as replacing the teams, that are homogeneous, by heterogeneous teams in order to share different contexts, know-how and knowledge. Concerning theory of knowledge creation, some results of studied cases made available the identification of eight observations that question some assumptions of this theory, as the high turnover or the guaranteed stability on work can perform on employees' commitment to knowledge creation.

Keywords: Knowledge management. Knowledge creation. Knowledge. Information. Environmental education. Environmental education programs. Universities.

Recebido em 25.11.2012 Aceito em 20.09.2014

\section{Introdução}

Nas últimas décadas, o conhecimento passou a ser considerado o principal recurso gerador de vantagem competitiva para as organizações a frente de outros recursos como a terra e o capital (DRUCKER, 1993; GARVIN, 1993; NONAKA, 1991, NONAKA, 1994; NONAKA; TAKEUCHI, 1997; STEWART, 1998). Com o conhecimento como principal ingrediente organizacional, faz-se necessário administrá-lo da maneira mais eficiente possível (NONAKA; TAKEUCHI, 1997; STEWART, 1998). Apesar de sua importância reconhecida, a gestão do conhecimento ainda não explorou profundamente seus processos, principalmente o processo de criação de novos conhecimentos (NONAKA, 1991; NONAKA, 1994; NONAKA; TAKEUCHI, 1997; NONAKA; TOYAMA, 2003). Com o objetivo de criar e utilizar da melhor forma esse recurso, a gestão do conhecimento tem sido estudada e aplicada em diversas áreas nos últimos anos.

Como consequência de um recente problema mundial - a degradação ambiental - a educação ambiental também tem se tornado um assunto crescente nos debates acadêmicos, políticos, empresariais e educacionais. No entanto, ela tem sido criticada por utilizar práticas pedagógicas desvinculadas da realidade, determinadas de forma autoritária de cima para baixo e negligenciar a gestão da informação e do conhecimento, elementos-chave para o desenvolvimento de um modelo sustentável (ALBAGLI, 1995; ALBUQUERQUE et al., 2011; DA CRUZ et al., 2013; GUIMARÃES, 1995; JACOBI, 2003; MAYOR, 1998; PEREIRA et al., 2013).

Em vista da grande preocupação com as questões ambientais na atualidade; do crescente papel da educação ambiental; da necessidade de se considerar a gestão da informação e do conhecimento no modelo de desenvolvimento sustentável; da necessidade de estudar o processo de criação do conhecimento em novos contextos; e em vista da gestão do conhecimento ter pouca aplicação em áreas que não sejam de empresas privadas na busca de lucratividade, é que se 
projeta a presente investigação. Nesse sentido, a pesquisa tem como objetivo principal estudar o processo de criação de novos conhecimentos em programas de educação ambiental.

No cenário atual, as instituições educacionais, em todos os níveis, apresentam um papel importante como instrumento de promoção de valores relacionados ao desenvolvimento sustentável. As universidades têm função da educação profissional e acadêmica e, também, a educação para a cidadania, para a convivência em comunidade, para o social e para o meio ambiente. Elas formam gestores e professores e têm ainda o importante papel de fonte geradora de conhecimento através da pesquisa científica, modificando atitudes e comportamentos no sentido de uma consciência ambiental e de uma responsabilidade ética.

[...] os trabalhos desenvolvidos dentro das instituições de ensino de nível superior têm um efeito multiplicador, pois cada estudante, convencido das boas ideias da sustentabilidade, influencia o conjunto, a sociedade, nas mais variadas áreas de atuação (KRAEMER, 2006, p.2).

Portanto, as universidades têm potencial formador de uma nova cultura compatível com o desenvolvimento sustentável. Paralelamente, elas geram grande quantidade de resíduos causando impactos consideráveis ao meio ambiente como consequência do elevado número de funcionários, professores e alunos. E, apesar disso, não se dirige muita atenção a elas nesse sentido, concentrando as causas dos danos ambientais principalmente às empresas privadas (ALBUQUERQUE et al., 2011; EVANGELINOS, 2009; FERRER-BALAS et al., 2009; JANTZ et al., 2013; KRAEMER, 2006; LUKMAN et al., 2009; PEREIRA et al., 2013; SIBBEL, 2009; STEPHENS et al., 2008; TOAKLEY, 2004; ZSÓKA et al., 2013).

A importância das atividades realizadas pelos programas de educação ambiental em instituições de ensino superior pode ainda ser demonstrada pelo levantamento realizado por Lipscomb et al. (2008) em universidades do Reino Unido. Os resultados demonstraram que intervenções extracurriculares têm impacto maior sobre as atitudes das pessoas que disciplinas e projetos curriculares. No entanto, esse tipo de intervenção tem sido pouco explorado por essas instituições; a constatação é que pouco recurso, tempo e dinheiro é dedicado a essas atividades (FERRER-BALAS et al., 2008; LIPSCOMB et al., 2008).

Sendo assim, serão objeto desse estudo os programas de educação ambiental de duas universidades localizadas no estado de São Paulo: a Universidade de São Paulo - USP, campus de São Carlos e a Universidade Estadual Paulista "Júlio de Mesquita Filho" - UNESP, campus de Bauru. As duas universidades têm grande representatividade no Brasil, na América Latina e até mesmo no mundo, integrando um seleto grupo de instituições de padrão mundial. Elas contribuem decisivamente para que o país seja visto com respeito nos cenários acadêmicos nacional e internacional. 
A USP é a maior universidade pública brasileira, considerada a universidade mais importante do país (LAM, 2011) e está classificada entre as universidades mais prestigiadas do mundo em diversos rankings (AQ ONLINE, 2011). Ela foi considerada a melhor universidade da América Latina e classificada dentre as 70 universidades com melhor reputação no planeta pela World University Rankings publicado pela revista Times Higher Education (AQ ONLINE, 2011; VEJA, 2012). Através do Webometrics Ranking of World Universities ela foi classificada novamente como a $1^{\text {a }}$ da América Latina e a $29^{a}$ do mundo (WEBOMETRICS RANKING OF UNIVERSITIES, 2014).

A UNESP foi classificada como a $9^{a}$ melhor universidade da América Latina e a $551^{\text {a }}$ de 600 universidades mundiais pelo QS World University Rankings de 2011 (O GLOBO, 2011). Bem como a $8^{a}$ do Brasil e a 328a do mundo Webometrics Ranking of World Universities (WEBOMETRICS RANKING OF UNIVERSITIES, 2014). E, a Times Higher Education apontou a Unesp como uma das 100 melhores universidades com menos de 50 anos existência do mundo e como a $82^{a}$ melhor instituição universitária dos países emergentes (REDAÇÃO ÉPOCA, 2013; GIANNINI, 2013).

\section{Teoria da criação do conhecimento de Nonaka}

Criação do conhecimento é um dos processos da gestão do conhecimento, por meio do qual as organizações adquirem, organizam e processam informação com o objetivo de gerar novos conhecimentos. É a ampliação do conhecimento criado pelos indivíduos (ALVARENGA NETO et al 2007; VON KROGH; ICHIJO; NONAKA, 2001).

Parte do reconhecimento da importância da gestão do conhecimento se deve a obra The Knowledge Creating Company, publicada em 1995 por Nonaka e Takeuchi. Dentre a literatura da gestão do conhecimento, a teoria da criação do conhecimento de Nonaka é uma das mais consolidadas atualmente (BRATIANU, 2010; HONG, 2011). Essa teoria parte do princípio de existência dos dois tipos de conhecimentos (explicito e tácito) apresentados por Polanyi (1983). O explícito é o conhecimento formal e sistemático, que pode ser registrado em papel, expresso em forma de frases ou desenhos, e por isso é comunicado e compartilhado facilmente. E o tácito é o conhecimento informal, vinculado aos sentidos, à percepção individual, à capacidade de expressão corporal, a convicções, a perspectivas, um conhecimento de difícil transmissão (NONAKA, 1991; VON KROG; ICHIJO; NONAKA, 2001) .

Polanyi é referência na área de gestão do conhecimento e tem sua definição conceitual de conhecimento consolidada e utilizada como base que fundamenta boa parte das teorias e modelos de gestão do conhecimento.

Segundo Nonaka e Takeuchi (1997), é necessário que se crie conhecimento organizacional, impedindo que o conhecimento seja mantido apenas no âmbito individual. É o indivíduo quem o cria e não a organização, no entanto, se essa informação não for transmitida à 
organização, mantendo-se somente em nível individual, ela será perdida. É nesse sentido que surge a importância do trabalho em equipe e das equipes interdisciplinares, pois indivíduos de diferentes áreas podem contribuir para a solução de problemas por meio de suas diferentes experiências de problemas anteriores e das resoluções específicas encontradas para cada um deles (RUS; LINDVALL, 2002).

Para que o conhecimento organizacional seja criado, é preciso converter os conhecimentos tácito e explícito dos indivíduos em conhecimento "amplificado" na organização. Esse processo de transformação do conhecimento é conhecido como modelo SECI de criação do conhecimento e ocorre através de quatro processos (NONAKA, 1991; NONAKA, 1994; NONAKA; TAKEUCHI, 1997; NONAKA; TOYAMA; KONO, 2000; TEE; LEE, 2013):

- Socialização: conversão de conhecimento tácito em conhecimento tácito, emprega processo de compartilhamento de experiências que envolvem trabalho em grupo e experiência prática e direta, gerando conhecimento compartilhado;

- Externalização: conversão de conhecimento tácito em conhecimento explícito, empreende ações que possam ser entendidas por outros por meio do diálogo, da reflexão coletiva e do uso de metáforas e analogias, gerando conhecimento conceitual;

- Combinação: conversão do conhecimento explícito em conhecimento explícito, utiliza processo de sistematização de conceitos construídos pelas equipes pela combinação, edição e processamento a fim de formar novo conhecimento, é a sistematização do conhecimento, gerando conhecimento sistêmico;

- Internalização: conversão do conhecimento explícito em conhecimento tácito, emprega processo de internalização do novo conhecimento explícito compartilhado na organização por meio do "aprender fazendo", da verbalização, dos modelos mentais e da diagramação, gerando conhecimento operacional.

- A organização deve oferecer um contexto apropriado que propicie e facilite a criação do conhecimento, o chamado Ba. O Ba é um contexto dinâmico e interativo que capacita o ambiente para a socialização dos membros da organização. Ele fornece a energia, a qualidade e o local para a conversão do conhecimento (NONAKA; TAKEUCHI, 1997, NONAKA; TOYAMA; KONNO, 2000; NONAKA; TOYAMA, 2003). Esse contexto organizacional pode ser físico (um escritório, uma sala, um espaço para negócios), virtual (teleconferência, email) e/ou mental (ideias, experiências compartilhadas) e é partilhado por dois ou mais indivíduos na organização 
(NONAKA; TAKEUCHI, 1997; NONAKA; KONO, 1998; NONAKA; NISHIGUSHI, 2001).

- Nonaka e Takeuchi (1997) complementam as condições para criar conhecimento na organização sugerindo três características-chave para a criação do conhecimento:

- O uso da linguagem figurada, da metáfora e da analogia para expressar intuições e insights, fazendo com que indivíduos fundamentados em diferentes contextos e com diferentes experiências compreendam algo intuitivamente por meio de símbolos, por exemplo, expressarem conhecimentos que possuem, mas não são capazes de dizer por palavras ou de maneira formal;

- O compartilhamento do conhecimento pessoal em conhecimento organizacional para que tenha sentido para outras pessoas e possa ser transmitido à organização toda na forma de discussões, diálogos e debates em grupo. "O que os líderes precisam fazer é conter e reter o conhecimento de forma que ele se torne propriedade da organização" (STEWART, 1998, p.90);

- O reconhecimento da relevância da ambiguidade e da redundância, pois estimulam o diálogo frequente e a comunicação, criando uma "base cognitiva comum" entre os funcionários, de onde nascem novos conhecimentos.

- Outro ponto da teoria afirma que a organização deve oferecer condições capacitadoras necessárias para que o indivíduo, no grupo, crie conhecimento organizacional, por meio de cinco condições:

- A intenção organizacional, definida como a aspiração de uma organização às suas metas, dissemina a visão, metas e objetivos da organização;

- A autonomia aos membros da organização introduz oportunidades inesperadas e possibilita a automotivação dos indivíduos para a criação de conhecimento;

- A flutuação e o caos criativo estimulam a interação entre a organização e o meio ambiente externo, possibilitando a exploração da ambigüidade, da redundância e dos ruídos dos sinais ambientais, aprimorando seu sistema de conhecimento;

- A redundância de informações transcendem as exigências operacionais imediatas dos membros da organização e aumentam o volume de informações a serem processadas podendo ocasionar o surgimento de novas ideias e;

- E a variedade de requisitos dos membros da organização que possibilita diferentes visões sobre uma mesma situação.

A criação do conhecimento organizacional envolve cinco fases: o compartilhamento do conhecimento tácito através da interação; a criação de conceitos por meio do diálogo e da reflexão coletiva; a justificativa de 
conceitos, uma espécie de filtragem das ideias; a construção de protótipos, transformando os conceitos em algo tangível; e o nivelamento do conhecimento, ou seja, a atualização contínua (NONAKA; TAKEUCHI, 1997; VON KROG; ICHIJO; NONAKA, 2001).

O conhecimento é inerente aos seres humanos, portanto, não se transfere ou compartilha com facilidade e espontaneidade. Existe um processo gerencial que facilita a criação e a difusão do conhecimento, constituindo-se em uma síntese dos dois modelos gerenciais dominantes top-down (de cima para baixo) e bottom-up (de baixo para cima) (ALVARENGA NETO et al 2007; NONAKA; TAKEUCHI, 1997). O modelo gerencial middle-up-down (do meio para cima e para baixo) coloca o gerente de nível médio no centro da gestão do conhecimento e redefine o papel da alta gerência e dos funcionários da linha de frente. Assim, a gerência de nível médio resolve a contradição entre o que a alta gerência espera criar (visões sonhadoras) e o que os funcionários de linha de frente realmente fazem (tarefas do mundo real) (NONAKA; TAKEUCHI, 1997).

Do mesmo modo que há um modelo gerencial que facilita a criação e a transmissão do conhecimento, há uma estrutura organizacional adequada. É a estrutura em hipertexto, combinação de dois tipos básicos de estrutura, a burocracia e a força-tarefa. Uma estrutura não-hierárquica e auto-organizada que funciona em conjunto com sua estrutura hierárquica formal. É um sistema aberto que interage com o ambiente externo (NONAKA; TAKEUCHI, 1997).

De acordo com a teoria da criação do conhecimento esses são os requisitos essenciais para uma organização criar novos conhecimentos.

\section{A informação e o conhecimento na educação ambiental}

A educação ambiental é "um aprendizado social baseado no diálogo e na interação, um processo de recriação e reinterpretação de informações, conceitos e significados, que podem se originar do aprendizado em sala de aula ou da experiência pessoal do aluno". Ela envolve conceitos, significados, aprendizado social, diálogo, interação, recriação e reinterpretação de informações (JACOBI, 2003, p.198). A própria definição de "educação ambiental" evidência sua relação com a teoria da criação do conhecimento e alguns de seus requisitos, por exemplo, os processos de socialização e externalização.

Soma-se a isso o fato de que a renúncia ambiental por parte das camadas sociais mais baixas da população pode ser explicada pelo baixo acesso a informações, dentre outros; por isso elas estão mais sujeitas aos riscos ambientais. A falta de informação e conhecimento desvaloriza as questões ambientais e leva à resistência e ao desinteresse por parte delas (LAYRARGUES, 2000). No entanto, apesar de diversos autores afirmarem a necessidade da educação ambiental trabalhar a informação e o conhecimento pertinente ao meio ambiente, poucos autores abordam o tema (JACOBI, 2003). 
É a partir da informação, do conhecimento e da percepção ambiental que as pessoas mudam seu comportamento e suas atitudes (AJZEN; FISHBEIN ${ }^{1}$, 1980 apud KUHLEMEIER et al., 1999; BRANDALISE et al., 2009; HUNGERFORD; VOLK ${ }^{2}, 1990$ apud POOLEY; O'CONNOR, 2000; PÁDUA; TABANEZ, 1998). Assim, a informação e o conhecimento são motores propulsores para a sensibilização e a identificação dos problemas ambientais e para a reflexão sobre a urgência da mudança dos atuais padrões de uso dos bens ambientais (RODRIGUES; COLESANTI, 2008).

Burgess et al. (1998) também relaciona o conhecimento ambiental às atitudes e ao comportamento ambiental (figura 1):

Figura 1 - Modelo de comportamento pró-ambiental

conhecimento
ambiental $\longrightarrow \begin{gathered}\text { atitude } \\ \text { ambiental }\end{gathered} \longrightarrow \begin{gathered}\text { comportamento } \\ \text { pró-ambiental }\end{gathered}$

Fonte: Burgess et al, 1998

Se o conhecimento ambiental é a entrada desse processo, como em qualquer processo, o input (conhecimento ambiental) deve ser processado (ou no caso, gerido) para que o resultado (output ou o comportamento pró-ambiental) seja alcançado. Esta descrição deixa clara a relação entre o conhecimento e a educação ambiental, bem como a importância de bem geri-lo.

Inclusive, o estudo sobre a relação do conhecimento com a atitude ambiental realizado por Bradley et al. (1999) com estudantes de segundo grau concluiu que as melhores atitudes ambientais estão direta e positivamente relacionadas aos grupos com maior conhecimento ambiental. Confirmando a importância dos programas de educação ambiental trabalharem o conhecimento que pretendem disseminar à comunidade.

Como é possível notar, argumentos de diversos autores explicitam a relação do conhecimento com a educação ambiental. Alguns autores ainda sugerem fatores necessários à educação ambiental, que se comparados a teoria de Nonaka, são justamente alguns dos requisitos apresentados na teoria da criação do conhecimento.

As experiências vividas têm maior influência sobre o comportamento das pessoas que as experiências informadas, pois aprende-se mais por meio de um processo de construção do conhecimento do que de um processo receptor de conhecimento pronto (CUNHA; OLIVEIRA , 2009; DIONYSIO; SANTOS, 2007; KOLLMUSS; AGYEMAN, 2002). Ou seja, o conhecimento tácito, o know-how e a socialização têm maior impacto

\footnotetext{
${ }^{1}$ AJZEN, I.; FISHBEIN, M. Understanding attitudes and predicting social behavior. Englewood Cliffs: Prentice Hall, 1980.

2 HUNGERFORD, H. R.; VOLK, T. L. Changing learner behavior through environmental education. Journal of Environmental Education, v.21, n.3, p.8-21, 1990.
} 
sobre as atitudes das pessoas do que a simples transmissão da informação.

Alguns autores sugerem um espaço no qual possam surgir as idéias para a construção de novos conhecimentos ambientais, pela experiência em grupo e pela troca de diferentes experiências, conhecimentos, maneiras de agir e de pensar (CUNHA; OLIVEIRA, 2009). Esse espaço sugerido por alguns autores que se dedicam ao estudo da sustentabilidade é similar ao Ba sugerido por Nonaka.

Além desse espaço, a área da educação ambiental exige uma área de trabalho em equipes multi, inter e pluridisciplinares, diálogo, trocas de ideias, e troca de ações (PERINOTTO; ZAINE, 2000). Estes fatores nada mais são do que os requisitos da teoria de Nonaka que incentivam a criação de novos conhecimentos.

Os argumentos apresentados nesta sessão demonstram a importância da informação e do conhecimento para a educação ambiental, o que consequentemente, implica em sua boa gestão e na proximidade da educação ambiental com a teoria da criação de novos conhecimentos, evidenciando a relevância do presente trabalho. A relação entre as duas áreas é apresentada no Quadro 1.

Quadro 1: similaridades entre a educação ambiental e a gestão do conhecimento

\begin{tabular}{|c|c|c|}
\hline \multicolumn{2}{|c|}{ Educação Ambiental } & Gestão do Conhecimento \\
\hline Conceito & Autor & Processo de Criação do Conhecimento \\
\hline - Informação ambiental & Jacobi (2003) & $\begin{array}{l}\text { - Recurso trabalhado pelo processo de criação } \\
\text { do conhecimento }\end{array}$ \\
\hline - Conhecimento ambiental & $\begin{array}{l}\text { Layrargues (2000) } \\
\text { Burgess, Harrison e Filius (1998) }\end{array}$ & $\begin{array}{l}\text { - Recurso trabalhado pelo processo de criação } \\
\text { do conhecimento }\end{array}$ \\
\hline - Aprendizado social & Jacobi (2003) & $\begin{array}{l}\text { - Socialização } \\
\text { - Externalização } \\
\text { - Internalização }\end{array}$ \\
\hline - Diálogo & $\begin{array}{l}\text { Jacobi (2003) } \\
\text { Perinotto e Zaine (2000) }\end{array}$ & $\begin{array}{l}\text { - Socialização } \\
\text { - Externalização } \\
\text { - Internalização }\end{array}$ \\
\hline - Interação & Jacobi (2003) & $\begin{array}{l}\text { - Socialização } \\
\text { - Externalização } \\
\text { - Internalização }\end{array}$ \\
\hline - Troca de experiência & Cunha e Oliveira (2009) & - Socialização \\
\hline $\begin{array}{l}\text { - Troca de ideias e maneiras } \\
\text { de pensar }\end{array}$ & $\begin{array}{l}\text { Perinotto e Zaine (2000) } \\
\text { Cunha e Oliveira (2009) }\end{array}$ & $\begin{array}{l}\text { - Socialização } \\
\text { - Externalização }\end{array}$ \\
\hline - Experiência pessoal & Cunha e Oliveira (2009) & $\begin{array}{l}\text { - Socialização } \\
\text { - Externalização }\end{array}$ \\
\hline - Troca de informação & Cunha e Oliveira (2009) & $\begin{array}{l}\text { - Externalização } \\
\text { - Combinação }\end{array}$ \\
\hline - Troca de conhecimento & Cunha e Oliveira (2009) & - Externalização \\
\hline - Vivencia de experiências & $\begin{array}{l}\text { Froehlich, Biassusi e Neuenfeldt } \\
\text { (2005) }\end{array}$ & - Externalização \\
\hline $\begin{array}{l}\text { - Troca de diferentes maneiras } \\
\text { de agir }\end{array}$ & Cunha e Oliveira (2009) & $\begin{array}{l}\text { - Externalização } \\
\text { - Internalização }\end{array}$ \\
\hline - Experiência grupal & Cunha e Oliveira (2009) & $\begin{array}{l}\text { - Externalização } \\
\text { - Internalização }\end{array}$ \\
\hline - Troca de ações & $\begin{array}{l}\text { Perinotto e Zaine (2000) } \\
\text { Cunha e Oliveira (2009) } \\
\end{array}$ & - Internalização \\
\hline - Equipes multidisciplinares & Perinotto e Zaine (2000) & $\begin{array}{l}\text { - Condição capacitadora: variedade } \\
\text { requisitos }\end{array}$ \\
\hline - Espaço para o surgimento da & Cunha e Oliveira (2009) & - $\mathrm{Ba}$ \\
\hline
\end{tabular}




\begin{tabular}{|c|c|c|}
\hline $\begin{array}{lll}\begin{array}{l}\text { construção } \\
\text { conhecimentos }\end{array} & \text { de novos } \\
\end{array}$ & & \\
\hline $\begin{array}{l}\text { - Acesso à informação } \\
\text { - Disseminação } \\
\text { conhecimento }\end{array}$ & Layrargues (2000) & $\begin{array}{l}\text { - Quinta fase do processo de criação do } \\
\text { conhecimento } \\
\text { - Disseminação do conhecimento }\end{array}$ \\
\hline $\begin{array}{l}\text { - Criação e produção de } \\
\text { conhecimentos e conceitos } \\
\text { - Recriação e reinterpretação } \\
\text { de informações }\end{array}$ & $\begin{array}{l}\text { Jacobi (2003) } \\
\text { Carta de Belgrado - Reigota (1994) } \\
\text { Di Giovanni (2005) }\end{array}$ & $\begin{array}{l}\text { - Segunda fase do processo de criação do } \\
\text { conhecimento } \\
\text { - Objetivo final do processo de criação do } \\
\text { conhecimento }\end{array}$ \\
\hline
\end{tabular}

Fonte: ESCRIVÃO et al, 2011

\section{Métodos de pesquisa}

A pesquisa pode ser caracterizada de natureza qualitativa, pois buscou perceber insights e conhecer o processo de criação do conhecimento, entender detalhadamente como ele ocorre nos programas estudados, sem a pretensão de buscar generalizações e padrões de comportamentos. Quantos aos fins, ela pode ser caracterizada como uma pesquisa descritiva, já que teve como objetivo primordial a descrição das características do objeto de estudo e de seus processos de criação do conhecimento. E quanto aos meios, foi realizado um estudo comparativo de casos, em razão do objetivo de conhecer detalhadamente e identificar características comuns e específicas do objeto de estudo e seus processos interativos de criação e transformação do conhecimento, mostrando como eles afetam e influenciam o funcionamento da organização, bem como a possível identificação de questões-chave para a pesquisa (COLLIS; HUSSEY, 2005; DENCKER; VIÁ, 2001; GIL, 1991; VERGARA, 1997).

A coleta de dados foi realizada através da entrevista com 0 responsável por cada programa e com os membros mais ativos, que podem ser considerados "elementos-chave" para o conhecimento das atividades realizadas pelo programa. As entrevistas foram guiadas por um roteiro desenvolvido a partir do protocolo da pesquisa. Com o intuito de verificar a estrutura e clareza do roteiro de entrevista formulado, foi realizado um estudo de caso-piloto no programa de educação ambiental da Universidade Federal de São Carlos, pelo qual as questões foram aperfeiçoadas (TRIVINOS, 1987; MANZINI, 1991; REA; PARKER, 2000³ apud BELEI; et al. 2008).

Cada programa de educação ambiental é formado por funcionários, professores e alunos de sua universidade. No caso da UNESP foram entrevistados dois docentes coordenadores, sendo um responsável pelas questões administrativas e o outro pelos projetos e atividades realizados; e os dois alunos bolsistas que atuavam diariamente em todas as atividades do programa. Na USP foram entrevistados o funcionário responsável pelo programa, dois coordenadores e quatro alunos bolsistas que desenvolviam os projetos e participavam de todas as atividades. Os alunos que participavam esporadicamente dos projetos não foram entrevistados. No total, onze entrevistas foram realizadas. Após a

${ }^{3}$ REA, L. M.; PARKER, R. A. Desenvolvendo perguntas para pesquisas. In: Metodologia de pesquisa: do planejamento à execução. São Paulo: Pioneira, p. 57-75, 2000. 
realização das entrevistas, estas foram transcritas e submetidas à aprovação de cada respondente como forma de aprimorar a fidedignidade, assegurando que as respostas obtidas sejam verdadeiras e não influenciadas por quaisquer condições (CAREGNATO; MUTTI, 2006).

A análise de dados foi realizada de acordo com a metodologia qualitativa, pautando-se principalmente nos procedimentos apresentados por Bardin (1977) e Ludke e André (1986). Primeiramente, foi realizado o que se costuma chamar de pré-análise, que incluiu a organização de todo o material, a divisão deste em partes e o relacionamento destas partes. Também se buscou fazer a identificação de tendências e padrões que pudessem ser relevantes frente às características especificas estudadas (LUDKE; ANDRÉ, 1986; MINAYO, 2008). Explicitado e sistematizado o conteúdo das mensagens, realizou-se a escolha das unidades de codificação. Os diferentes elementos foram classificados em categorias descritivas segundo critérios suscetíveis de fazer surgir um sentido capaz de introduzir certa ordem na confusão inicial. A finalidade dessa classificação foi, a partir desses índices, deduzir certas informações. Optou-se por analisar as entrevistas por tema e categoria, que, no caso, eram as próprias categorias da pesquisa.

De acordo com o objetivo proposto, as categorias da pesquisa foram definidas conforme apresentadas no Quadro 2:

Quadro 2 - Categorias da Pesquisa

\begin{tabular}{|c|c|c|}
\hline CATEGORIA PRINCIPAL & SUBCATEGORIA & OPERACIONALIZAÇĀO \\
\hline \multirow{21}{*}{$\begin{array}{l}\text { Processo de criação do } \\
\text { conhecimento }\end{array}$} & \multirow{4}{*}{ Processo SECI } & Socialização \\
\hline & & Externalização \\
\hline & & Combinação \\
\hline & & Internalização \\
\hline & \multirow[t]{3}{*}{ Características-chave } & $\begin{array}{l}\text { Transformação de conhecimento individual em } \\
\text { organizacional (diálogos, discussões e debates em } \\
\text { grupo) }\end{array}$ \\
\hline & & Linguagem figurada e simbolismo \\
\hline & & Ambiguidade e redundância \\
\hline & \multirow{4}{*}{ Condições capacitadoras } & Intenção organizacional \\
\hline & & Autonomia \\
\hline & & Flutuação e caos criativo \\
\hline & & Variedade de requisitos \\
\hline & \multirow{5}{*}{ Fases para se criar conhecimento } & Compartilhamento \\
\hline & & Criação do conceito \\
\hline & & Justificativa do conceito \\
\hline & & Construção do arquétipo \\
\hline & & Difusão interativa \\
\hline & \multirow[t]{2}{*}{ Contexto apropriado $\mathrm{Ba}$} & Ba físico \\
\hline & & Ba virtual \\
\hline & Modelo gerencial & Middle-up-down \\
\hline & Estrutura hierárquica & Em hipertexto \\
\hline & Disseminação do conhecimento & $\begin{array}{l}\text { Disseminação em todas as direções por toda a } \\
\text { organização }\end{array}$ \\
\hline
\end{tabular}

Fonte: Dados da Pesquisa

Quanto à escolha dos casos selecionados, a opção realizada se pautou: 
- Na importância das universidades escolhidas, considerando-se o sistema educacional, acadêmico e de pesquisa;

- No impacto que elas causam no meio ambiente, por estarem entre as maiores universidades do país;

- E na comparação com outros programas de educação ambiental de universidades da região em contato prévio, a qual revelou que os programas selecionados são os mais ativos, elaborados e sistematizados, possibilitando um estudo mais estruturado.

\section{Análise dos dados}

Após a análise dos dados com a técnica de análise conteúdo, as semelhanças e, principalmente, as diferenças entre a teoria da criação do conhecimento e os programas de educação ambiental estudados puderam ser observadas. Primeiramente, percebeu-se que os principais pontos fracos dos dois programas são os mesmos:

- A socialização do conhecimento entre os membros dos programas ocorre frequentemente, mas a socialização entre os membros e os usuários (alunos, professores e funcionários) é fraca. As entrevistas demonstraram que a socialização do conhecimento fica comprometida devido a estabilidade funcional dos servidores docentes e técnicos-administrativos (já que ambos os programas são de universidades públicas) e pela rotatividade dos alunos que sabem que estão apenas de passagem pela universidade. Dessa maneira, os usuários mostram baixo comprometimento com os objetivos e atividades dos programas estudados ou porque têm sua estabilidade garantida ou porque estão somente de passagem pela universidade;

- O processo de combinação de conhecimento é outro ponto fraco, já que os programas não fazem uso de ferramentas que possibilitem o armazenamento e a busca de informações e conhecimentos, bem como de ferramentas que facilitem a interação entre membros e usuários;

- A internalização é, dos quatro processos de transformação, o menos explorado. Segundo os relatos dos entrevistados isso ocorre pela falta de verba e tempo. Segundo a teoria, se os conhecimentos que foram socializados, externalizados e combinados não forem internalizados pela prática, o ciclo não será completado e novos conhecimentos não serão criados. Dessa forma, é de extrema importância criar atividades do tipo "aprender fazendo", caso contrário, toda a energia, recursos e tempo dedicado aos três processos anteriores (socialização, externalização e combinação) não serão, de fato, incorporados; 
- Não há consciência da importância da variedade de requisitos, consequentemente, as atividades são realizadas em grupos homogêneos ou por categorias (professores, funcionário, alunos) e ou por cursos. A formação dos grupos para a realização das atividades deve ser feita de maneira que cada grupo possua pessoa das diferentes categorias, dos diferentes cursos e dos diferentes anos;

- Não há espaço físico e nem virtual que possibilite a interação entre os membros e usuários. O espaço físico existente se restringe a utilização por parte dos membros. É essencial a existência de um espaço físico e um espaço virtual interativo que atraiam os usuários para participarem do programa e interagirem entre si.

Esses pontos, se explorados a partir da perspectiva da gestão e criação do conhecimento, podem proporcionar uma melhor gestão dos conhecimentos referentes aos programas, bem como a criação de novos conhecimentos relacionados às atividades e práticas ambientais, consolidando experiências e aprendizados junto aos membros e usuários dos programas.

\section{Resultados e discussão}

Como resultados da pesquisa pôde-se sugerir algumas práticas aos Programas de Educação Ambiental possibilitando que eles possam gerir melhor seu conhecimento, bem como criar novos. Além disso, alguns fatos observados e analisados geraram uma reflexão quanto a necessidade de relativizar o uso dos conceitos da teoria da criação do conhecimento.

\subsection{Sugestões aos programas de educação ambiental}

A análise das práticas dos programas de educação ambiental, fundamentada nas entrevistas realizadas, em comparação com as categorias conceituais da pesquisa, a saber, os requisitos da teoria da criação do conhecimento, gerou sugestões práticas aos Programas de Educação Ambiental sintetizadas no Quadro 3:

Quadro 3 - Sugestões aos programas de EA

\begin{tabular}{|c|l|}
\hline VARIÁVEIS & \multicolumn{1}{c|}{ SUGESTŐES } \\
\hline Socialização & $\begin{array}{l}\text { Realizar reuniões, almoços, encontros gastro-filosóficos, eventos culturais, palestras, propiciando } \\
\text { o compartilhamento de experiências }\end{array}$ \\
\hline Externalização & $\begin{array}{l}\text { Fazer uso de figuras, imagens, filmes, músicas, fluxogramas, gráficos, maquetes para transmitir } \\
\text { conceitos e ideias }\end{array}$ \\
\hline Combinação & $\begin{array}{l}\text { Fazer uso de bases de dados, relatórios, arquivos, manutenção de criações e experiências em } \\
\text { livros e apostilas, pesquisas para sistematização dos conceitos e ideias. }\end{array}$ \\
\hline Internalização & $\begin{array}{l}\text { Realizar atos dinâmicos como a plantação de mudas, oficinas de construção de composteira, } \\
\text { oficinas de produção de objetos com material reciclado, feira da barganha (troca e venda de } \\
\text { produtos usados) que possibilitem a incorporação do conhecimento explicito de forma tácita }\end{array}$ \\
\hline $\begin{array}{c}\text { Transformação de } \\
\text { conhecimento individual em } \\
\text { organizacional }\end{array}$ & $\begin{array}{l}\text { Realizar as atividades sempre em grupo para que o conhecimento não se mantenha em âmbito } \\
\text { individual somente }\end{array}$ \\
\hline $\begin{array}{c}\text { Linguagem figurada e } \\
\text { simbolismo }\end{array}$ & $\begin{array}{l}\text { Fazer uso de linguagem figurada, metáfora, simbolismo exemplos para transmitir o } 0 \\
\text { conhecimento e as ideias aos outros }\end{array}$ \\
\hline
\end{tabular}




\begin{tabular}{|c|c|}
\hline Ambiguidade e redundância & $\begin{array}{l}\text { Proporcionar mais de uma maneira de realizar cada atividade sempre que possível gerando } \\
\text { informações que transcendem as exigências operacionais imediatas dos membros da } \\
\text { organização, aumentando o volume de informações a serem processadas; e possibilidade de } \\
\text { realizar cada atividade mais de uma vez, de onde nascem novos conhecimentos ao estimular o } \\
\text { diálogo frequente e a comunicação e ao criar uma "base cognitiva comum" entre as pessoas }\end{array}$ \\
\hline Intenção organizacional & $\begin{array}{l}\text { Criar uma cultura que se preocupe em trabalhar o conhecimento constantemente, que busque } \\
\text { adquirir e criar novos conhecimentos e passar isso aos membros do programa }\end{array}$ \\
\hline Autonomia & $\begin{array}{l}\text { Os membros do programa devem sentir não somente que podem opinar, mas também devem ter } \\
\text { suas colocações e ideias levadas em consideração na tomada de decisões, o que introduz } \\
\text { oportunidades inesperadas e possibilita a automotivação dos indivíduos para a criação de } \\
\text { conhecimento }\end{array}$ \\
\hline Flutuação e & $\begin{array}{l}\text { Propiciar mudanças e um ambiente que não seja totalmente previsível, estimulando a interação } \\
\text { entre a organização e o meio ambiente externo, possibilitando a exploração da ambiguidade, da } \\
\text { redundância e dos ruídos dos sinais ambientais, aprimorando seu sistema de conhecimento }\end{array}$ \\
\hline Variedade de $r$ & $\begin{array}{l}\text { Os grupos não devem ser separados por categorias e depois por cursos como costumam fazer os } \\
\text { programas, os grupos devem ser heterogêneos, num mesmo grupo deve haver alunos de } \\
\text { diversos cursos, de graduação, pós-graduação, funcionários e docente, para que diferentes } \\
\text { contextos, experiências, conhecimentos, culturas sejam compartilhados }\end{array}$ \\
\hline Compartilhar & Compartilhar o conhecimento tácito através da interação \\
\hline Criação do co & Criar conceitos através do dialogo e da reflexão coletiva \\
\hline Justificativa do & $\begin{array}{l}\text { Realizar uma espécie de filtragem para justificar os conceitos viáveis e que devam ser levados a } \\
\text { diante }\end{array}$ \\
\hline Construção c & Construir protótipos para tornar os conceitos em algo tangível \\
\hline Difusão interativa & Atualização contínua \\
\hline Ba físico & $\begin{array}{l}\text { Um espaço físico para que os membros possam passar um tempo juntos, possam compartilhar } \\
\text { momentos, realizar atividades juntos é essencial para a criação de novos conhecimentos, bem } \\
\text { como para que os usuários possam comparecer, visitar }\end{array}$ \\
\hline Ba virtual & $\begin{array}{l}\text { Do mesmo modo, um espaço virtual interativo, que forneça a comunicação e a interação entre os } \\
\text { membros e entre estes e os usuários, também possibilita essa vantagem, assim, mais do que um } \\
\text { simples espaço virtual, é necessário que esse espaço seja interativo e não apenas informativo }\end{array}$ \\
\hline $\begin{array}{l}\text { Modelo gerencial } \\
\text { middle-up-down } \\
\text { Estrutura hierárquica } \\
\text { em hipertexto }\end{array}$ & $\begin{array}{l}\text { Comunicação informal, relações pessoais, flexíveis e alteradas com certa frequência para que um } \\
\text { membro não execute sempre as mesmas tarefas e sempre do mesmo modo facilitam a estrutura } \\
\text { em hipertexto e o modelo organizacional middle-up-down sugeridos pela teoria da criação do } \\
\text { conhecimento }\end{array}$ \\
\hline $\begin{array}{l}\text { Disseminação do } \\
\text { conhecimento }\end{array}$ & 0 \\
\hline
\end{tabular}

\subsection{Reflexões sobre a teoria da criação do conhecimento}

A análise dos dados coletados demonstrou que alguns dos requisitos apresentados por Nonaka como necessários a gestão e criação do conhecimento não ocorrem em determinados contextos e ambientes ou se comportam de maneira diferente. Alguns requisitos que o autor afirma ser essencial para a gestão e criação de novos conhecimentos não pareciam ser tão necessários para os programas estudados. Dessa forma, os casos estudados possibilitaram algumas observações à teoria da criação do conhecimento de Nonaka.

1 Analisando os casos estudados, no que diz respeito ao processo de socialização, nota-se que os programas de educação ambiental não têm legitimidade e poder para convencer os usuários (alunos da universidade, da creche e das escolas públicas da região, docentes e funcionários da universidade) a modificarem suas atitudes. A socialização ocorre mais frequentemente entre os membros internos do programa, mas de forma 
muito fraca externamente, entre os membros e os usuários. Diferente do que acontece no ambiente empresarial estudado por Nonaka, onde as grandes corporações têm poder e legitimidade para intimar ou convencer seus clientes e fornecedores. As entrevistas sugerem que isso ocorre devido a dois fatores: a estabilidade garantida por parte dos funcionários e professores que são concursados e; a rotatividade alta por parte dos alunos que estão na universidade apenas de passagem. Esses fatores contribuem para o baixo comprometimento com o programa, dificultando a socialização do conhecimento. Assim, a socialização nem sempre ocorre facilmente como demonstra a teoria da criação do conhecimento, ela depende do poder e da legitimidade que a organização tem sobre seus membros e stakeholders (usuários/cliente/fornecedores), como demonstra a figura 2 .

Figura 2 - Influencia do poder e da legitimidade da organização sobre os stakeholders no processo de socialização

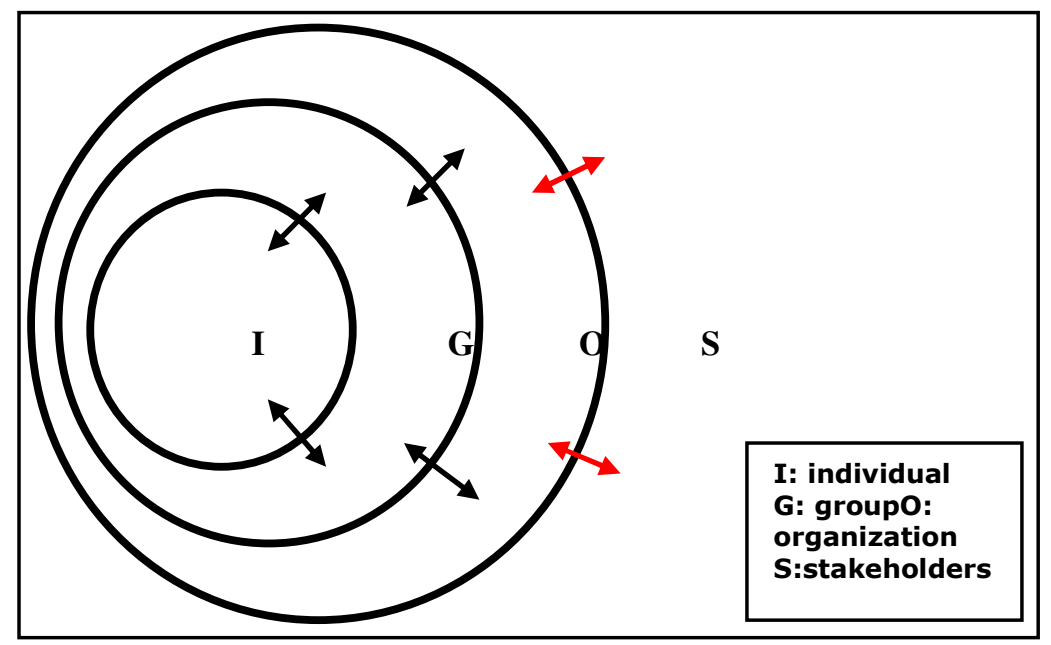

Fonte: Dados da Pesquisa

2 Dependendo da natureza da organização, o alinhamento entre os objetivos da organização (no caso, os programas de educação ambiental) com os clientes e fornecedores (no caso, os usuários) pode ser fraco. No ambiente de grandes corporações estudado por Nonaka, já existe um ambiente de cooperação e os interesses dos negócios levam a um alinhamento dos objetivos entre as partes. Isso não pode ser generalizado para qualquer ambiente. Na universidade os interesses são divergentes, os professores, alunos possuem e funcionários possuem interesses diferentes. Assim, sem a existência de um alinhamento entre os objetivos das diversas partes, é difícil para os programas "convencer" os usuários a participarem das atividades.

3 Soma-se a isso o fato das organizações estudadas por Nonaka possuírem diversas medidas de desempenho e recompensa que incentivam o trabalho em grupo, a socialização e a criação de novos 
conhecimentos, as quais não existem nos programas, e possivelmente em outros tipos de organizações como uma pequena empresa.

4 A analogia é apresentada na teoria da criação do conhecimento como algo simples, que ocorre facilmente. Para que o indivíduo faça uso de analogias é necessário que ele tenha uma sofisticação intelectual e muitas vezes, como no caso dos programas estudados, os funcionários possuem um conhecimento técnico e especializado e não o conhecimento intelectual mais generalista que a execução da analogia exige. Os programas estudados revelaram grande dificuldade por parte das pessoas, na maioria das vezes com formação mais técnica e especialista, em fazer uso da analogia.

5 Os casos estudados demonstraram que a redundância é outro fator difícil de implantar na organização. Ela está ligada a cultura da organização. No ambiente estudado por Nonaka, onde reina a cultura da inovação, é simples inserir a redundância das práticas e atividades da organização. Mas nos programas estudados, a cultura demonstrou ser uma barreira à tentativa de inserção da redundância. É provável que esse fato esteja presente em organizações tradicionais, por exemplo, e organizações baseadas na produção em massa.

6 Enquanto as empresas citadas na teoria trabalham com uma visão de estratégia, na qual o produto busca explorar um novo segmento de negócio, movimentando toda a organização; os programas, e possivelmente outros tipos de organizações como as pequenas empresas, precisam trabalhar de forma imediatista e operacional. Dessa forma, a quarta (desenvolvimento de um protótipo ou teste) das cinco fases do processo de criação do conhecimento se mostrou inviável no caso dos programas estudados.

$7 \mathrm{O}$ Ba como um contexto criativo, é algo que já existe e é facilmente inserido em um ambiente corporativo caracteristicamente desafiador e competitivo, como o estudado pela teoria da criação do conhecimento. Nos programas estudados, as entrevistas demonstraram que faltam recursos e cobranças para que isso ocorra. Acredita-se que em outros tipos de organizações, inseridas em ambientes diferentes do estudado por Nonaka essa dificuldade também possa estar presente.

8 A teoria apresenta a informalidade como uma característica do modelo e da estrutura organizacional, os quais são requisitos para a criação de novos conhecimentos. Nesse estudo, percebeu-se que a informalidade nem sempre é fator que, junto de outros, possibilita a criação do conhecimento. Depende da "fonte" dessa informalidade. No caso de grandes empresas estudadas por Nonaka, essa informalidade é resultado de uma estrutura organizacional horizontalizada, com muitos especialistas, onde há flexibilidade para que estes se movimentem, gerando a interação, a troca de conhecimentos e as experiências necessárias à criação de novos conhecimentos. Já nos programas de educação ambiental, a informalidade advém da simplicidade da estrutura e do tamanho pequeno das organizações, não estando assim, relacionado 
com a flexibilidade e com a interação com o contexto de formalidade, metas diversas e acirrada competitividade.

Todas essas considerações construídas sobre os casos estudados revelam que os requisitos apresentados como necessários à gestão e criação de novos conhecimentos, pela teoria da criação do conhecimento de Nonaka, muitas vezes, não se aplicam a toda e qualquer tipo de organização. É preciso considerar as diferentes naturezas dos diversos tipos de organizações. A ideia de um modelo único que represente as diversas organizações não é concebível atualmente. Para a abordagem contingencial da teoria das organizacões, é necessário entender que existem diferentes organizações, com naturezas e características específicas e, que essas especificidades requerem diferentes ações. Portanto, essa pesquisa pôde perceber que alguns dos pressupostos da teoria da criação do conhecimento, apesar de sua grande contribuição, não atingem o resultado esperado nas organizações estudadas. Dessa forma, esses pressupostos devem ser relativizados, sendo necessária uma aplicação (do modelo de criação do conhecimento) diferenciada e adequada às contingências das organizações. Tomada em perspectiva normativa, prescritiva, o modelo da criação do conhecimento pode ser ineficiente ou prejudicial.

\section{Considerações Finais}

Essa pesquisa apresenta dois resultados principais.

Em primeiro lugar, sugere práticas que favorecem a gestão e a criação de novos conhecimentos aos programas de educação ambiental. Estes programas têm muito a melhorar sua efetividade com a adoção de requisitos conceituais já divulgados pela teoria da criação do conhecimento, e aqui traduzidos para a realidade ambiental. Como sugestões, os programas podem realizar eventos que propiciem o compartilhamento de conhecimento e experiência; fazer uso de bases de dados, relatórios e instrumentos que possibilitem armazenar conhecimento; realizar atividades práticas ensinadas em palestras e aulas para incorporar o conhecimento tácito de maneira explícita; promover o trabalho em grupos multidisciplinares, dentre outros. Trabalhar a construção de uma cultura organizacional favorável a criação do conhecimento e explorar as ambiguidades e redundâncias dos temas também pode favorecer a gestão e criação de novos conhecimentos. Além disso, disponibilizar um espaço físico aos membros do programa de forma que possam compartilhar momentos e realizar atividades em equipe $e$, incentivar a existência de um espaço virtual interativo, que forneça a comunicação e a interação entre os membros e os usuários é essencial para a criação de novos conhecimentos.

Como segundo resultado, a pesquisa identifica algumas lacunas na teoria da criação do conhecimento e apresenta o entendimento de que nem todos os seus pressupostos funcionam para toda e qualquer organização. Os casos estudados demonstraram que devido a 
especificidades organizacionais, alguns requisitos podem não favorecer a gestão e criação de novos conhecimentos, assim os requisitos devem ser relativizados em sua aplicação. Já a teorização da criação do conhecimento precisa levar em conta aspectos contingenciais importantes à validade de seus conceitos. Primeiro, a socialização do conhecimento, em organizações que não sejam empresas, ressente-se da falta de comprometimento ou da fluidez de seu público. Em segundo, em sequência ao tema anterior, a legitimidade do mercado torna o objetivo empresarial um alvo consensual, o que não ocorre com facilidade na universidade com segmentos e correntes de ideias bastante heterogêneos. Em terceiro, o uso intelectualmente atraente (e belo) da analogia pesa como uma utopia, mesmo em segmentos da universidade. Quanto a redundância sempre foi tratada pela administração como desperdício, desde que Taylor declarou que os trabalhadores faziam cera para trabalhar menos; imagine a dificuldade que ainda se terá para vencer este imaginário ligado à eficiência. Quinto, as empresas de Nonaka claramente trabalham visando o longo prazo, a estratégia é o veículo que leva do presente ao futuro. Os programas, como a maioria das pequenas empresas, trabalham o presente, são refém do operacional. Sexto, a cultura da criatividade e a prática da inovação não são possíveis, ou mesmo necessárias, como na maioria as pequena empresas; assim, o $\mathrm{Ba}$ aparece como um conceito mistificador e sem sentido. Por último, a informalidade na teoria das corporações é o oásis no deserto da formalidade, ou seja, a informalidade é uma ocorrência no contexto da formalidade, da estruturação e do controle tecnológico sofisticado; na pequenez dos programas estudados, e das pequenas empresas, a informalidade é a realidade, é a estruturação possível, é a precariedade da objetivação dos controles.

Pesquisas complementares são necessárias para investigar a ocorrência desses pressupostos em outros tipos de organizações e novas investigações precisam ser feitas em pequenas empresas, em organizações do terceiro setor, em outros segmentos do setor público, e mesmo em universidades a fim de detalhar as conclusões aqui alcançadas.

\section{Referências}

ALBAGLI, S. Novos espaços de regulação na era da informação e do conhecimento. In: LASTRES, H.M.M.; LABAGLI, S. Informação e globalização na era do conhecimento. Rio de Janeiro: Campus, 1995, p.290-313.

ALBUQUERQUE, N. H. C.; MARQUES, C. C.; LIRA, J. P. A.; VASCONCELOS, A. L. M.; VASCONCELOS, S. C. S.; ARAUJO, I. F.; BARBOSA, E. A. Environmental education and its influence in an Institution of Higher Education: the study of workers of the university restaurant in the city of Campina Grande, Brazil. In: INTERNATIONAL SYMPOSIUM ON OCCUPATIONAL SAFETY AND HYGIENE, Anais..., p.427-431, 2011. 
ALVARENGA NETO, R.C.D.; BARBOSA, R.R.; PEREIRA, H.J. Gestão do conhecimento ou gestão de organizações da era do conhecimento? Um ensaio teórico-prático a partir de intervenções na realidade brasileira. Perspectivas em Ciência da Informação, Belo Horizonte, v.12, n.1, p.5-24, jan./abr. 2007.

AQ ONLINE. University of São Paulo Ranks Among Top Global Schools. TIMES HIGHER EDUCATION, AMERICA QUARTERLY, 7 out. 2011. Disponível em: http://americasquarterly.org/node/2936. Acesso em: 04 set. 2014.

BARDIN, L. Análise de conteúdo. Lisboa: Edições 70, 1977.

BELEI, R. A.; GIMENIZ-PASCHOAL, S. R.; NASCIMENTO, E. N.; MATSUMOTO, P. H. V. R. O uso de entrevista, observação e vídeogravação em pesquisa qualitativa. Cadernos de Educação, v.30, p.187199, 2008.

BURGESS, J.; HARRISON, C.; FILIUS, P. Environmental communication and the cultural politics of environmental citizenship. Environment and Planning, London, v.30, n.8, p.1445-1460, 1998.

BRANDALISE, L.T. et al. A Percepção e o comportamento ambiental dos universitários em relação ao grau de educação ambiental. Gestão e Produção, v.16, n.2, p.273-285, abr./jun, 2009.

BRADLEY, J.C.; WALICZEK, T.M.; ZAJICEK, J.M. Relationship between environmental knowledge and environmental attitude of high school students. Journal of Environmental Education, Madison, v.30, n.3, p.1721, spring 1999.

BRATIANU, C. A Critical Analysis of Nonaka's Model of Knowledge Dynamics. Electronic Journal of Knowledge Management, v.8, n.2, p.193$200,2010$.

CAREgnATO, R. C. A.; MUTTI, R. Pesquisa qualitativa: análise de discurso versus análise de conteúdo. Texto Contexto Enfermagem, v.4, n.15, p.679-684, out./dez. 2006.

COLLIS, J.; HUSSEY, R. Pesquisa em administração: um guia prático para alunos de graduação e pós-graduação. Porto Alegre: Bookman, 2005.

CUNHA, M.T.; OLIVEIRA, R.C.S. Programa de construção do conhecimento em educação ambiental. 2009.2 Disponível em: http://www.minerva.uevora.pt/hiflex/curso/ue/activi1.htm. Acesso em: 21 mai. 2009.

DA CRUZ, E. R.; MARIANO, Z. F.; CARDOSO, J. K. A. Diagnóstico da educação ambiental e geografia no ensino médio. Revista Eletrônica Itinerarius Reflectionis, v.1, n.14, 2013.

ZSÓKA, A.; SZERÉNYI, Z. M.; SZÉCHY, A.; KOCSIS, T. Greening due to environmental education? Environmental knowledge, attitudes, consumer behavior and everyday pro-environmental activities of Hungarian high 
school and University student. Journal of Cleaner Production, v.48, p.126138, 2013.

DENCKER, A.F.M.; VIÁ, S.C. Pesquisa empírica em ciências humanas: com ênfase em comunicação. São Paulo: Futura, 2001.

DIONYSIO, R.C.C.; SANTOS, F.C.A. Evolução da informação apoiadora da gestão ambiental: uma análise centrada em seus estágios evolutivos e nos agentes decisórios. Informação e Informação, Londrina, v.12, n.2, p.1-11, jul./ago. 2007.

DRUCKER, P. Sociedade pós-capitalista. São Paulo: Pioneira, 1993.

ESCRIVÃO, G.; NAGANO, M. S.; ESCRIVÃO FILHO, E. A gestão do conhecimento na educação ambiental. Perspectivas em Ciência da Informação, v.16, n.1, p.92-110, 2011.

REDAÇÃO ÉPOCA. Brasil tem 4 universidades entre as melhores dos países emergentes. ÉPOCA, 04 dez. 2013. Disponível em: http://epoca.globo.com/vida/noticia/2013/12/brasil-tem-b4-universidadesb-entremelhores-dos-paises-emergentes.html. Acesso em: 31 ago. 2014.

EVANGELINOS, K. I.; JONES, N.; PANORIO, E. M. Challenges and opportunities for sustainability in regional universities: a case study in Mityleni, Greece. Journal of Cleaner Production, v.17, p.1154-1161, 2009.

LAM, C. USP e Unicamp estão na lista de melhores universidades do mundo. EXAME, 05 set. 2011 Disponível em: http://exame.abril.com.br/carreira/noticias/usp-e-unicamp-estao-na-lista-demelhores-universidades-do-mundo. Acesso em: 03 set. 2014.

FERRER-BALAS, D.; ADACHI, J.; BANAS, S.; DAVIDSON, C. CI.; HOSHIKOSHI, A.; MISRHA, A.; MOTODOA, Y.; ONGA, M.; OSTWALD, M. An international comparative analysis of sustainability transformation across seven universities. International Journal of Sustainability in Higher Education, v.9, n.3, p.295-316, 2008.

FERRER-BALAS, D.; BUCKLAND, H.; MINGO, M. Explorations on the University's role in society for sustainable development through a systems transition approach: case study of the Technical University of Catalonia. Journal of Cleaner Production, v.17, p.1075-1085, 2009.

FROEHLICH, E.; BIASSUSI, M.; NEUENFELDT, C.R. Educação ambiental na construção do conhecimento e na preservação do meio ambiente: uma ferramenta de interação extensão rural - escola - comunidade. Cerro Grande do Sul, 2005.2 Disponível em: http://www.biodiversidade.rs.gov.br/arquivos/1161519748Educacao ambiental na construcao do conhecimento e na preservacao do MA.pdf. Acesso em: 26 mai. 2010.

GARVIN, D.A. Building a learning organization. Harvard Business Review, New York, v.71, n.4, p.93-102, jul./ago. 1993. 
GIANNINNI, M. J. A UNESP no ranking das economias emergentes. ESTADÃO, 9 dez. 2013. Disponível em: http://educacao.estadao.com.br/noticias/geral,artigo-a-unesp-no-ranking-daseconomias-emergentes,1106372. Acesso em: 05 set. 2014.

GIL, A.C. Como elaborar projetos de pesquisa. São Paulo: Atlas, 1991. GUIMARÃES, M. A dimensão ambiental na educação. Campinas: Papirus, 1995.

HONG, J. F. L. Glocalizing Nonaka's knowledge creation model: issues and challenges. Management Learning, v.43, n.2, p.199-215, 2011.

JACOBI, P. Educação ambiental, cidadania e sustentabilidade. Cadernos de Pesquisa, São Paulo, n.118, p.189-205, mar, 2003.

JANTZ, A. R.; TOMIO, D.; UBER, D.; APPEL, G.; WEBER, S. Uma proposta de temas geradores para Educação Ambiental em escolas de comunidades com vulnerabilidade ambiental. Revista Eletrônica do Mestrado em Educação Ambiental, v.30, n.2, p.335-355, jul./dez. 2013.

KOLLMUSS, A., AGYEMAN, J. Mind the gap: why do people act environmentally and what are the barriers to proenvironmental behavior? Environmental Education Research, v.8, n.3, p.239-260, 2002.

KRAEMER, M. E. P. O ensino universitário e o desenvolvimento sustentável. 2006. Disponível em: http://www.comscientianimad.ufpr.br/2006/02/acervo cientifico/outros artigos/artigo maria elizabeth.pdf. Acesso em: 29 ago. 2010.

KUHLEMEIER, H.; VAN DEN BERGH, H.; LAGERWEIJ, N. Environmental knowledge, attitudes, and behavior in Dutch secondary education. The Journal of Environmental Education, Madison, v.30, n.2, p.4-14, winter, 1999.

LAYRARGUES, P.P. Educação para a gestão ambiental: a cidadania no enfrentamento político dos conflitos socioambientais. In: LOUREIRO, C.F.B. (Org.). Sociedade e meio ambiente: a educação ambiental em debate. São Paulo: Cortez, 2000, p.85-155.

LIPSCOMB, B. P.; BUREK, C. V.; POTTER, J. A.; RIBCHESTER, C.; DEGG, M. R. An overview of extra-curricular education for sustainable development interventions in UK universities. International Journal of Sustainability in Higher Education, v.9, n.3, p.222-234, 2008.

LUDKE, M.; ANDRÉ, M. E. D. A. Pesquisa em educação: abordagens qualitativas. São Paulo: EPU, 1986.

LUKMAN, R.; KRAJNC, D.; GLAVIC, P. Fostering collaboration between universities regarding regional sustainability initiatives, the University of Maribor. Journal of Cleaner Production, v.17, p.1143-1153, 2009.

MANZINI, E. J. A entrevista na pesquisa social. Didática, São Paulo, v.26/27, p.149-158, 1991. 
MAYOR, F. Preparar um futuro viável: ensino superior e desenvolvimento sustentável. In: CONFERÊNCIA MUNDIAL SOBRE O ENSINO SUPERIOR. TENDÊNCIAS DE EDUCAÇÃO SUPERIOR PARA O SÉCULO XXI. Anais da Conferência Mundial do Ensino Superior. Paris, 1998.

MINAYO, M.C.S. O Desafio do conhecimento: pesquisa qualitativa em saúde. São Paulo: Hucitec, 2008.

NONAKA, I. The Knowledge-creating company. Harvard Business Review, New York, v.69, n.6, p.96-104, nov./dez. 1991.

A Dynamic theory of organizational knowledge creation. Organization Science, Linthicum, v.5, n.1, p.14-37, 1994.

NONAKA, I.; TAKEUCHI, H. Criação de conhecimento na empresa. Rio de Janeiro: Campus, 1997.

NONAKA, I.; KONNO, N. The Concept of "Ba": building a foundation for knowledge creation. California Management Review, Berkeley, v.40, n.3, p.40-54, spring, 1998.

NONAKA, I.; TOYAMA, R.; KONNO, N. SECI, Ba and Leadership: a unified model of dynamic knowledge creation. Long Range Planning, Oxford, v.33, n.1, p.5-34, 2000.

NONAKA, I.; NISHIGUSHI, T. Knowledge emergence: social, technical and evolutionary dimensions of knowledge creation. New York: Oxford University Press, 2001.

NONAKA, I.; TOYAMA, R. The Knowledge-creating theory revisited: knowledge creating as synthesizing process. Knowledge Management Research and Practice, v.1, n.1, p.2-10, jul. 2003.

O GLOBO. USP lidera ranking das 200 melhores universidades da América Latina divulgado pela QS, empresa especializada em educação. O GLOBO, 04 out. 2012 Disponível em: http://oglobo.globo.com/sociedade/educacao/usplidera-ranking-das-200-melhores-universidades-da-america-latina-divulgado-pela-qsempresa-especializada-em-educacao-2787947. Acesso em: 31 ago. 2014.

OLIVEIRA JÚNIOR, M.M. Competitividade baseada no conhecimento. In: CAVALCANTI, M. (Org.). Gestão estratégica de negócios: evolução, cenários, diagnóstico e ação. São Paulo: Thompson Learning, 2007, p.121-152.

PÁDUA, S.; TABANEZ, M. Educação ambiental: caminhos trilhados no Brasil. São Paulo: Ipê, 2008.

PEREIRA, C. C.; SILVA, F. K.; RICKEN, I.; MARCOMIN, F. E. Percepção e Sensibilização Ambiental como instrumentos à Educação Ambiental. Revista Eletrônica do Mestrado em Educação Ambiental, v.30, n.2, p.86106, jul./dez. 2013.

PERINOTTO, J.A.J.; ZAINE, M.F. Educação ambiental: estratégias e ações para a construção da cidadania. In: FREITAS, M.I.C.; LOMBARDO, M.A. 
Universidade e comunidade na gestão do meio ambiente. Rio Claro: AGETEO- UNESP, UCENPARCERIAS-UNESP - Universidade de Auburn, 2000 , p. 170.

POOLEY, J.A.; O'CONNOR, M. Environmental education and attitudes: emotions and beliefs are what is needed. Environment and Behavior, v.32, n.5, p.711-723, set. 2000.

POLANIY, M. The tacit dimension. Massachusetts: Peter Smith, Gloucester,1983.

REIGOTA, M. O Que é educação ambiental. São Paulo: Brasiliense, 1994.

RODRIGUES, G.S.S.C.; COLESANTI, M.T.M. Educação ambiental e as novas tecnologias de informação e comunicação. Sociedade e Natureza, Uberlândia, v.20, n.1, p.51-66, jun. 2008.

RUS, I.; LINDVALL, M. Knowledge management in software engineering. IEEE Software, v.19, n.3, p.26, mai./jun/ 2002.

SIBBEL, A. Pathways towards sustainability through higher education. International Journal of Sustainability in Higher Education, v.10, n.1, p.68$82,2009$.

STEPHENS, J. C.; HERNANDEZ, M. E.; ROMÁN, M.; GRAHAM, A. C.; SCHOLZ, R. W. Higher education as a change agent for sustainability in different cultures and contexts. International Journal of Sustainability in Higher Education, v.9, n.3, p.317-338, 2008.

STEWART, T. A. Capital intelectual. Rio de Janeiro: Campus, 1998.

TEE, M. Y.; LEE, S. S. Advancing understanding using Nonaka's model of knowledge creation and problem-based learning. Computer-Supported Collaborative Learning, v.8, pp.313-331, 2013.

TOAKLEY, A. R. Globalization, sustainable development and universities. Higher Education Policy, v.17, n.3, p.311-324, 2004.

TRIVINOS, A. N. S. Introdução à pesquisa em ciências sociais: a pesquisa qualitativa em Educação. São Paulo: Atlas; 1987.

VEJA. USP está entre 70 universidades com melhor reputação do mundo. VEJA, 15 mar. 2012. Disponível em: http://veja.abril.com.br/noticia/educacao/usp-esta-entre-70-universidades-commelhor-reputacao-do-mundo. Acesso em: 04/09/2014.

VERGARA, S.C. (1997). Projetos e relatórios de pesquisa em administração. São Paulo: Atlas, 1997.

VON KROGH, G.; ICHIJO, K.; NONAKA, I. Facilitando a criação de conhecimento: reinventado a empresa com o poder da inovação. Rio de Janeiro: Campus, 2001.

WEBOMETRIC RANKING UNIVERSITIES. 2014. Disponível em: http://www.webometrics.info/en/Latin America/Brazil. Acesso em: 02 set. 2014 